\title{
High-Content Analyses of Vaccinia Plaque
}

\section{Formation}

\section{Artur Yakimovich* and Jason Mercer*}

* Corresponding authors

\section{Summary}

Vaccinia virus plaque assays are employed for quantification of virus titer through serial dilution of virus on a monolayer of cells. Once the virus titer is diluted enough to allow for only few cells of the monolayer to be infected, clonal spread of infection can be detected by observing the lesion in the cell monolayer or using virus specific staining methods. Beyond simple titration, plaque formation bares priceless underlying information about subtle virus-host interactions and their impact on virus spread during multiple rounds of infection. These include virus infectivity, the mode of virus spread, virus replication rate and spatiotemporal spread efficacy. How this underlying information can be harnessed using a high-content imaging setup is discussed here.

Keywords: Vaccinia, Virus plaque assay, virus spread, high-content imaging 


\section{Introduction}

The plaque assay is one of the earliest quantitative methods developed in Virology. Originally developed for bacteriophages, the assay was later adapted for mammalian viruses by Renato Dulbecco in $1953(\mathbf{1 , 2})$. In a monolayer of producer cells cultured in a dish, viruses form plaques by first infecting only a few cells of the monolayer. These initial infected cells replicate and spread infection to their neighbors by cell-tocell $(3,4)$ or cell-free transmission in a lytic or non-lytic fashion $(5-7)$ eventually forming clonal populations of infected cells within the monolayer. Ultimately, cytopathic effect, infected cell migration (8) and cell lysis (5) form a lesion - an empty spot in the cultured cell monolayer. This empty spot is termed a plaque. Plaques can sometimes be seen by the naked eye but are more commonly visualized upon fixation and staining of the cell monolayer with crystal violet. The number of plaques corresponds directly to the number of fully infectious plaque forming units (PFUs) in the virus inoculum.

In a broader sense, a viral plaque may refer to the resulting lesion - the end point of plaque formation - but also to a distinguishable clonal population of infected cells. The latter can be visualized using a virus that expresses a fluorescent transgene or immunocytochemistry combined with fluorescence microscopy of the infected monolayer $(\mathbf{9}, \mathbf{1 0}, 5)$. While the majority of replication competent viruses form plaques in favorable conditions, the mechanisms of plaque formation vary significantly, depending on the mode of virus transmission, time post infection and state of the host cell and the culture medium (11). 
In the aftermath of this complexity, viral plaques are often different sizes and shapes, carrying a footprint of the underlying host-pathogen interactions that have occurred $(\mathbf{1 0}, 5,11,9)$. In a liquid culturing medium, viruses that spread via cell-cell contact form round plaques, whereas cell-free spread of viruses results in plaques that are typically elongated (comet-shaped) as a result cell-free virus particles being subject to passive mass transfer in the liquid medium $(\mathbf{5 , 1 1})$. The formation of comet-shaped plaques can be prevented by the addition of low-melt agarose, carboxymethylcellulose (12) or other gelling agents to the culture medium. This may, however, conceal important hostpathogen interactions manifesting in these phenotypes, including the amount of cellfree virus egressing from the initially infected cell, cell-free virus infectivity, the relationship between cell-cell and cell-free spread etc. $(\mathbf{1 0 , 1 1 , 1 3 ) . ~ I n s t e a d ~ o f ~ a r t i f i c i a l l y ~}$ preventing these phenotypes from occurring it is possible to apply computer vision based quantitative analysis combined with model-based fluid dynamics analysis to untangle the complex shapes that viral plaques take. To understand virus spread dynamics this approach can be further combined with live-cell time-lapse imaging and cell tracking $(10,11,14,15)$.

VACV spreads through a monolayer of cells using a combination of cell-cell and cellfree mechanisms. Cell-cell spread is mediated by cell-associated enveloped virions (CEVs), which after egress remain tethered to the cell surface. Cell-free spread is directed by extracellular enveloped virions (EEVs) which are formed when CEVs are released from the infected cell surface $(\mathbf{1 6 , 1 7 , 1 0 )}$. Cell-free VACV spread can also be mediated through intracellular mature virions (IMVs) released upon cell lysis. VACV

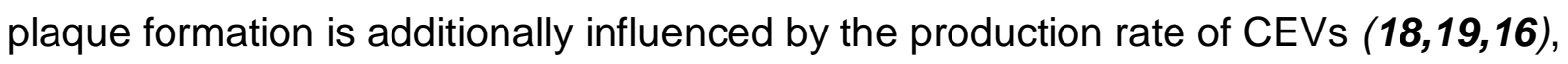

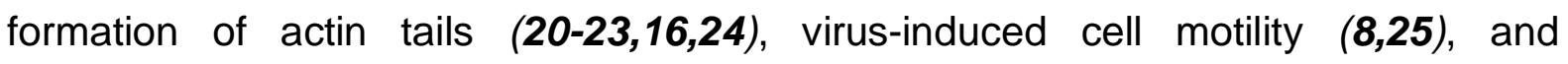


superinfection repulsion $(26,9)$. In light of this complexity, classical plaque assays yield limited mechanistic information regarding specific plaque phenotypes. High-content functional (e.g. fluorescence microscopy) and label-free (e.g. phase-contrast microscopy) microscopy-based plaque assays, in turn, can unveil information about mode of spread (cell-cell or cell-free, lytic or pre-lytic $(\mathbf{1 0 , 1 3 )})$, stage of infection (Early or late VACV promoter based transgenes (27)), host cell state or monolayer dynamics employing time-lapse microscopy (9). This readily quantifiable imaging data allows for

measurement of plaque features like: signal intensity, size, shape, directionality, and growth dynamics which can then be correlated to important biological defining hostpathogen interactions. In this chapter we describe how to set-up, perform and analyze VACV plaque formation under live cell conditions in a high-content fashion.

\section{Materials}

\subsection{Endpoint High-content Plaque Assay}

1. Depending on the scale: 96-well or 384-well imaging grade micro-titer plates (see Note 1, 2 and 3).

2. Tissue culture cells (see Note 4).

3. Culture medium: Dulbeco's Minimal Essential Medium (DMEM) containing 10\% $\mathrm{v} / \mathrm{v}$ fetal calf serum (FCS) (see Note 5 ).

4. Phosphate buffered saline (PBS).

5. $0.25 \%$ Trypsin/EDTA.

6. Wild type VACV stock (see Note 6).

7. Infection medium: DMEM without supplements.

8. Fixation solution: $4 \% \mathrm{w} / \mathrm{v}$ Paraformaldehyde (PFA) solution in PBS.

9. Quenching solution: $50 \mathrm{mM} \mathrm{NH}_{4} \mathrm{Cl}$ in PBS. 
10. Permeabilization solution: $0.1 \%$ Triton® X-100 in PBS.

11. Blocking buffer: $5 \% \mathrm{w} / \mathrm{v}$ bovine serum albumin (BSA) in PBS.

12. Heochst 33342 nuclear dye and other stains (see Note 7).

13. Primary antibody against viral protein, diluted in 5\% BSA in PBS at an appropriate concentration.

14. Secondary antibody conjugated to a fluorescent dye (e.g. Alexa488) raised to recognize the species of the primary antibody.

\subsection{Time-lapse High-content Plaque Assay}

1. Depending on the scale: 96 -well or 384 -well imaging grade micro-titer plates (see Note 1, 2 and 3).

2. Tissue culture cells ( see Note 4).

3. Culture medium: Dulbeco's Minimal Essential Medium (DMEM) containing 10\% v/v fetal calf serum (FCS) (see Note 5).

4. Infection medium: DMEM without supplements.

5. Live-imaging medium (see Note 8).

6. Phosphate buffered saline (PBS).

7. $0.25 \%$ Trypsin/EDTA.

8. Fluorescent recombinant VACV stock (see Note 9).

9. Deionized water for humidity control.

\section{Methods}

\subsection{High-content Plaque Assay Imaging}

1. Move the confluent indicator cell culture dish into the laminar safety cabinet. 
2. Wash the cells with PBS.

3. Detach the cells by incubating in $0.25 \%$ Trypsin/EDTA for $3-5 \mathrm{~min}$ at $37^{\circ} \mathrm{C}$.

4. Count cells using either automated cell counter or manual cell counting chamber.

5. Dilute the cell suspension to the desired concentration with cell culture medium in multi-channel pipette reservoir (see Note 10).

6. Re-suspend cells in the reservoir and dispense them into the micro-titer plate at desired volume using either automated or manual multi-channel pipette (see Note 11).

7. To ensure an even distribution of the cells throughout the growth surface leave cells standing at room temp for 20-40 min before transferring them into the incubator.

8. Culture cells over night at $37^{\circ} \mathrm{C}$ in a humidified cell culture incubator.

9. One day after cell seeding verify homogeneous monolayer formation using inverted transmission light microscope.

10. Prepare warm virus stock and infection medium to $37^{\circ} \mathrm{C}$ (see Note 12 ).

11. If serial pre-dilution prior to inoculum dispensing into the plate is required perform it as outlined in Fig. 1a or perform the serial dilution of the virus inoculum directly in the plate as outline in Fig. 1b and step 12 of this protocol (see Notes 13 and 14).

12. To perform a serial dilution in the plate using multi-compartment reservoir add an appropriate amount of fresh infection medium into the compartments or reservoirs, making sure medium volume in the wells of the multi-titer plate corresponds to the desired dilution factor (see Fig 1a, b and Note 13).

13. Using a multi-channel pipette dilute the first step in the group of wells of the plate designated for the first step (e.g. column 1, Fig 1b). 
14. Mix the added inoculum with the medium in the well by aspirating and dispensing $3 x$.

15. Exchange the pipette tips (see Note 14).

16. From the first step column using a multi-channel pipette transfer the amount of liquid appropriate to the dilution factor to the next column and so on (see Note 15 and 16 and Fig. 1b).

17. Incubate for the desired time at $37^{\circ} \mathrm{C}$ or other temperature required by experimental conditions.

18. For endpoint imaging proceed to step 19. For time-lapse imaging proceed to Subheading 3.2.

19. After $12-24 \mathrm{~h}$ of incubation, fix the plate by removing the culture medium and adding fixation solution (see Note 17).

20. Incubate between 30 and 60 min on the bench at room temperature (see Note 18).

21. Wash cells $3 x$ with PBS.

22. Incubate cells at RT in quenching solution for $5 \mathrm{~min}$.

23. Incubate cells at RT in permeabilization solution for 10 min (see Note 19).

24. Incubate cells at RT in blocking buffer for 30-60 min.

25. Stain samples with primary and secondary antibodies according to respective staining protocols.

26. Stain for host cell nuclei using Hoechst or other nuclear dye (see Note 7).

27. Upon staining imaging of the plate using an automated high-content microscope can be performed. For this choose the appropriate magnification and number of fields of view, wells desired, as well as, fluorescence channels in the microscope settings (see Note 20). 
28. Acquire and store the image data in an automated fashion and export the images upon acquisition (see Note 21 and Fig. 1c).

29. Once acquired and exported in "TIF" format, move image data to the storage location desired for analysis by Plaque2.0 software.

\subsection{Time-lapse High-content Plaque Assay}

1. Ensure the necessary incubation parameters (required temperature, $\mathrm{CO}_{2}$ and humidity) are possible and switched on at the microscope at least one hour before imaging.

2. Proceeding from Subheading 3.1 step 17 live-cell image the plate immediately or after a desired period of time using an automated high-content microscope (see Note 20, Note 24, Note 25 and Fig. 1D).

3. After desired period of incubation (typically $24 \mathrm{~h}$ ), fix cells for additional endpoint analyses (proceed to Subheading 3.1, step 19), or dispose of virus infected cells according to established safety protocols.

4. Images should exported and stored as single time point per folder (a single time point is inclusive of multiple field-of-view positions), where each position and channel is stored as an individual "TIF" file (see Note 26).

\subsection{High content analysis using Plaque 2.0}

1. Download Plaque2.0 from http://plaque2.github.io/ website.

2. Once downloaded, follow the installations instructions on the website (see Note 22).

3. Once installed, start the software and set up the "Main Parameters" (see Fig. 2) according to the location of your data and the preferred location and name of analysis results. 
4. In case the full well picture has been obtained in tiled acquisition of several sites (fields of view) per well, stitching of the image tiles is required. To perform stitching, check the box next to the "Stitch" pane and set up respective parameters (see Fig. 2a and Table 1).

5. Use "Test Settings" button, to ensure parameters are set correctly. It may make sense to run this module before running the rest of the analysis.

6. In case the stitched outside-of-the-well area (circular wells) is present in the square field of view of the image, to improve the precision of the analysis these parts of the image can be masked. For this, activate the check box in the "Mask" pane and set up the masking parameters (see Fig. 2B and Table 1).

7. Use "Test Settings" button, to ensure parameters are set correctly.

8. If nuclear stain was used in the assay to visualize total cells in the well, activated "Monolayer" pane and set up the nuclear detection parameters (including thresholding strategy etc.) in the respective window (see Fig. 3A and Table 1).

9. Use "Test Settings" button, to ensure parameters are set correctly.

10. To detect VACV plaques in the $\mathrm{HCl}$ data, activate the "Plaque" pane. Set relevant parameters, including plaque signal threshold (see Fig. 3B and Table 1; Note 23).

11.Prior to starting the analysis use "Test Settings" button, to ensure parameters are set correctly.

12. Once parameters are set and tested press "Run" button to run the analysis on the whole folder of images. Once complete, results will be saved as comma separated value (CSV) files in the folder selected.

13. For time-lapse acquisition data, repeat the analysis on each folder containing single time point data per folder (see Subheading 3.2, step 4). 
14. After analyses, locate results consisting of two CSV files. The first CSV file contains data relevant to the image-based features readouts (i.e. number of plaques, number of cells in the monolayer; see Table 2, for the full list of imagebased features and their detailed explanation). The second CSV file contains individual plaque based features (i.e. plaque size and shape; number of infected cells in the plaque; see Table 2 for the full list of plaque-based readouts and their detailed explanation).

15. Correlate the plaque objects using their coordinates (see Note 26).

\section{Notes}

1. In case the assay is performed in fluorescence microscopy setting, to lower the background coming from the reflection of the stray excitation light black-walled imaging plates are used. Imaging quality microtiter plates have flat clear bottom, with thickness ranging between 0.17 (so called \#1.5 thickness) $\mathrm{mm}$ and 0.64 $\mathrm{mm}$ (\#4 thickness).

2. Material and coating of the plates bottom play and important role for cell adhesion, typically plates sold for tissue culture are coated Material, evenness and thickness of the plates bottom may have an impact on the quality of the images obtained in the automated microscopy, especially if autofocusing is used. Altogether, microscopic imaging plates should be chosen based on experimental conditions, experimental scale, imaging hardware and other specific requirements. As a rule-of-thumb, thin mineral coverslip glass bottom plates give a better signal-noise in fluorescence imaging, as well as, autofocusing performance, however require a better coating for cell adhesion and are significantly more expensive. 
3. Plate bottom coating is of key importance for good cell adhesion and, depending on the nature of the indicator cells used (concerning their properties like contact inhibition, readiness to grow in a monolayer), may be crucial to the formation of a homogenous monolayer. A homogeneous monolayer is, in turn, crucial to obtain reproducible results in plaque assay. Assure the bottom of the plates are made of either organic (polystyrene or cyclic olefin co-polymer) or mineral coverslip glass.

4. Plaque assay indicator cell line often refers to the cell the virus is typically grown in, as they need to be able to support virus replication, as well as, be highly susceptible to the virus infection. We routinely use BSC40 or HeLa cells, but one can use any cell line as long as they can be grown to confluency and infected by VACV. Depending on the experimental setup dictated by the goal of the study or visualization modality different cells may be used.

5. To prevent the spread of cell-free viral particles in the cell culture medium through advection a gelling agent may be added. E.g. this can be ultra-low melting cell culture grade agarose cell at final concentration of 0.5 to $3 \%(\mathbf{1 3})$.

6. Visualization strategies for VACV include (but are not limited to) use of immunofluorescent (IF) staining against viral proteins and recombinant viruses expressing fluorescent proteins. Since IF visualization is possible only in an endpoint assay upon fixation this protocol is focusing on IF and wild type VACV (VACV). However, endpoint plaque assays can also be performed with fluorescent recombinant VACVs. While VACV strain Western Reserve produces circular plaques, e.g. VACV strain International Health Department J (VACV IHD-J) produces comet shaped plaques (27). Both strains may be used in this assay. 
7. While nuclear staining is not strictly necessary for the high-content plaque assay, it may be very informative to evaluate e.g. total cell count and other parameters of the system. Since some nuclear dyes may inhibit VACV infection (27), nuclear staining here is only included in the endpoint assay post fixation.

8. Live imaging compatible medium typically doesn't contain phenol red, since the latter may significantly increase background fluorescence and lead to uneven background throughout the duration of the longer time-lapse acquisition, due to $\mathrm{pH}$ changes. Additionally, it is important to choose a suitable buffering agent for live imaging culture medium. E.g. HEPES is often used to buffer medium for short-term incubations.

9. Recombinant VACV expressing fluorescent proteins (e.g. VACV encoding GFP under early promoter) or chimeric fluorescent proteins (conjugated with viral proteins) may be used here. The main criteria of selection here are signal-tonoise (the higher the better) in the imaging system used and signal uniformity in the infected cell (the more uniform the better).

10. It is advisable to seed the right number of cells to ensure highest possible confluence. This way a close tight and homogeneous monolayer may be achieved on the morning after seeding. E.g. a good range to get a monolayer of BSC40 cells in a 96-well plate overnight is between 5,000 and 50,000 cells per well (depending on the cultivation conditions, cell line passage etc.). At low dispensing volumes and while working with hydrophobic surfaces, it is important to ensure medium contact with the plate bottom during dispensing.

11. It is important to have sufficient medium volume in the well to ensure homogenous cell seeding. E.g. for a typical 96-well plate a minimum 
recommended volume is $50 \mu \mathrm{L}$. However, higher volume is preferred for better reproducibility.

12. Upon thawing the frozen VACV aliquot it is important to sonicate it to ensure precise titration results.

13. Serial dilution of the virus can be performed either prior to dispensing the inoculum into the plate (pre-dilution) or directly in the plate (Fig. 1a, b). The choice between the two depends on the viral titre of the stock virus solution and area of the well in the plate used.

14. As virus particles may become trapped even by low-adhesive plastic surfaces, it is important to plan liquid handling to ensure as little as possible change of vessels. At the same time, since adsorption of virus particles on plastic surfaces is reversible, it is important to exchange tips between dilution steps, to ensure titration precision.

15. Add the inoculum into the first dilution and proceed transferring volume (according to the chose dilution factor, e.g. 1/10 or $1 / 2$ of total) from one dilution into subsequent in a step wise manner. Make sure to exchange the disposable pipettes or tips upon each dilution (Fig. 1a).

16. E.g. in a 96 -well plate for $1 / 10$ or $1 / 2$ factors it is convenient to have 90 or 50 $\mu \mathrm{L}$ respectively.

17. Alternatively, fixation can be performed by diluting a higher percentage PFA solution directly in the culture/infection medium to arrive at $4 \%$ final PFA concentration. In such case the inoculum medium is not removed.

18. Longer fixation times are important to minimize the loss of cells in the monolayer upon washing.

19. Alternative permeabilization agents such as methanol or saponin can be used. 
20. Depending on the average plaque size and time of fixation, typically $2 x$ to $10 x$ magnification is used to image VACV plaques. To obtain a representative titer typically imaging of a complete well is desired. To date $\mathrm{SCMOS}$ chip sizes allow imaging a full 96 -well plate well in one image at $2 x$ magnification. E.g. using $4 x$ magnification on many systems a full 96-well plate well may be acquired in 4 separate images (fields of view), which can be stitched together in the imaging processing step. Increasing magnification and number of the fields of view increases acquisition time and requirements on storage and analysis.

21. If the data is saved in a proprietary format, make sure individual channels and fields of view are exported in "TIF" format for further analysis. This can be done using either the proprietary software of the high-content microscope or using the open source Fij//mageJ software.

22. To use the latest version of the Plaque2.0 software we suggest using the MATLAB $^{\text {TM }}$ (Mathworks) source code. For this download the source code folder. Download and install the MATLAB ${ }^{\mathrm{TM}}$ (Mathworks) integrated development environment (IDE). Start the IDE and open the source code folder. In the IDE run the PlaqueGUI.m file appropriate for your platform.

23. To ensure the plaques related signal is treated equally throughout the dataset global manual threshold is used. This parameter can be set interactively, by opening the threshold selection tool button next to the value input. Apart from thresholding and detection of putative plaques centers, the algorithm of Plaque2.0 software performs plaque area detection separating merging plaques using parameters defined in the optional "Fine detection" section (Fig. $3 b$ and Table 1). 
24. Live-cell imaging of VACV plaques typically requires a fluorescent reporter protein to be expressed by VACV assayed. However other live staining options may be possible.

25. Make sure time it takes to acquire all required wells in the plate isn't longer than the required time interval.

26. Correlation of the individual plaques may be performed in a downstream analysis, by associating respective positions of the plaque centers. This can be performed outside of the Plaque2.0 software (e.g. using Python or KNIME software (28)). A good starting point for such analysis is to correlate the plaques centers coordinates using the Nearest Neighbor algorithm within a restricted neighborhood comparable to the size of a plaque (10). 


\section{References}

1. D'Herelle F (1926) The bacteriophage and its behavior. The Williams \& Wilkins Company, Baltimore, Md.

2. Dulbecco R, Vogt M (1953) SOME PROBLEMS OF ANIMAL VIROLOGY AS STUDIED BY THE PLAQUE TECHNIQUE. Cold Spring Harbor Symposia on Quantitative Biology 18:273-279.

3. Mothes W, Sherer NM, Jin J, Zhong P (2010) Virus Cell-to-Cell Transmission. Journal of Virology 84:8360-8368.

4. Sattentau Q (2008) Avoiding the void: cell-to-cell spread of human viruses. Nature reviews Microbiology 6:815-826.

5. Yakimovich A, Gumpert H, Burckhardt CJ, Lütschg VA, Jurgeit A, Sbalzarini IF, et al. (2012) Cell-free transmission of human adenovirus by passive mass transfer in cell culture simulated in a computer model. Journal of virology 86:10123-10137.

6. Burckhardt CJ, Greber UF (2009) Virus movements on the plasma membrane support infection and transmission between cells. PLoS pathogens 5 (11):e1000621

7. Bär S, Daeffler L, Rommelaere J, Nüesch JP (2008) Vesicular egress of non-enveloped lytic parvoviruses depends on gelsolin functioning. PLoS pathogens 4 (8):e1000126

8. Sanderson CM, Way M, Smith GL (1998) Virus-induced cell motility. Journal of virology 72 (2):1235-1243

9. Doceul V, Hollinshead M, van der Linden L, Smith GL (2010) Repulsion of superinfecting virions: a mechanism for rapid virus spread. Science (New York, NY) 327:873-876.

10. Yakimovich A, Andriasyan V, Witte R, Wang I-H, Prasad V, Suomalainen M, Greber UF (2015) Plaque2. 0-A High-Throughput Analysis Framework to Score Virus-Cell Transmission and Clonal Cell Expansion. PloS one 10 (9):e0138760

11. Yakimovich A, Yakimovich Y, Schmid M, Mercer J, Sbalzarini IF, Greber UF (2016)

Infectio: a Generic Framework for Computational Simulation of Virus Transmission between Cells. mSphere 1 (1):e00078-00015

12. Russell WC (1962) A sensitive and precise plaque assay for herpes virus. Nature 195 (4845):1028-1029

13. Yakimovich A, Gumpert H, Burckhardt CJ, Lutschg VA, Jurgeit A, Sbalzarini IF, Greber UF (2012) Cell-free transmission of human adenovirus by passive mass transfer in cell culture simulated in a computer model. J Virol 86 (18):10123-10137.

14. Sbalzarini IF, Koumoutsakos P (2005) Feature point tracking and trajectory analysis for video imaging in cell biology. Journal of Structural Biology 151:182-195.

15. Tinevez J-Y, Perry N, Schindelin J, Hoopes GM, Reynolds GD, Laplantine E, Bednarek SY, Shorte SL, Eliceiri KW (2017) TrackMate: An open and extensible platform for single-particle tracking. Methods 115:80-90

16. Smith GL, Vanderplasschen A, Law M (2002) The formation and function of extracellular enveloped vaccinia virus. Journal of General Virology 83 (12):2915-2931

17. Moss B (2013) Poxviridae. In: Fields BN, Knipe DM, Howley PM et al. (eds) Fields

Virology, vol 1.6 edn. Lippincott Williams \& Wilkins, a Wolters Kluwer business, Philadelphia, USA, p 2664

18. Condit RC, Moussatche N, Traktman P (2006) In a nutshell: structure and assembly of the vaccinia virion. Advances in virus research 66:31-124 
19. Roberts KL, Smith GL (2008) Vaccinia virus morphogenesis and dissemination. Trends in microbiology 16 (10):472-479

20. Blasco R, Sisler J, Moss B (1993) Dissociation of progeny vaccinia virus from the cell membrane is regulated by a viral envelope glycoprotein: effect of a point mutation in the lectin homology domain of the A34R gene. Journal of virology 67 (6):3319-3325

21. Cudmore S, Cossart P, Griffiths G, Way M (1995) Actin-based motility of vaccinia virus. 22. Frischknecht F, Moreau V, Röttger S, Gonfloni S, Reckmann I, Superti-Furga G, Way M (1999) Actin-based motility of vaccinia virus mimics receptor tyrosine kinase signalling. Nature 401 (6756):926-929

23. McIntosh A, Smith GL (1996) Vaccinia virus glycoprotein A34R is required for infectivity of extracellular enveloped virus. Journal of Virology 70 (1):272-281

24. Wolffe EJ, Weisberg AS, Moss B (1998) Role for the vaccinia virus A36R outer envelope protein in the formation of virus-tipped actin-containing microvilli and cell-to-cell virus spread. Virology 244 (1):20-26

25. Valderrama F, Cordeiro JV, Schleich S, Frischknecht F, Way M (2006) Vaccinia virusinduced cell motility requires F11L-mediated inhibition of RhoA signaling. Science 311 (5759):377-381

26. Doceul V, Hollinshead M, Breiman A, Laval K, Smith GL (2012) Protein B5 is required on extracellular enveloped vaccinia virus for repulsion of superinfecting virions. Journal of General Virology 93 (Pt 9):1876-1886

27. Yakimovich A, Huttunen M, Zehnder B, Coulter $\amalg$, Gould V, Schneider C, Kopf M, McInnes CJ, Greber UF, Mercer J (2017) Inhibition of poxvirus gene expression and genome replication by bisbenzimide derivatives. Journal of Virology 91 (18):e00838-00817

28. Fillbrunn A, Dietz C, Pfeuffer J, Rahn R, Landrum GA, Berthold MR (2017) KNIME for reproducible cross-domain analysis of life science data. Journal of biotechnology 261:149156 


\section{Figures}

a

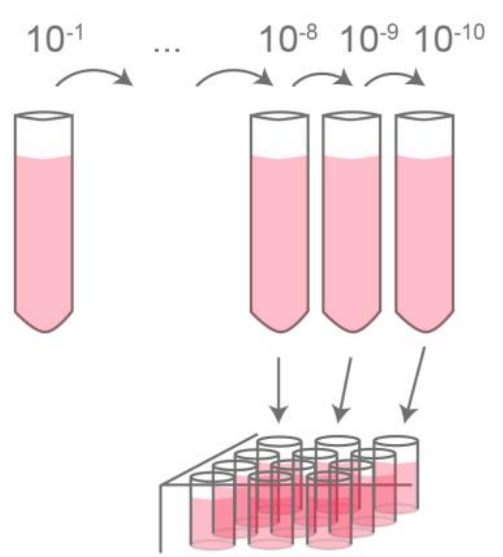

C

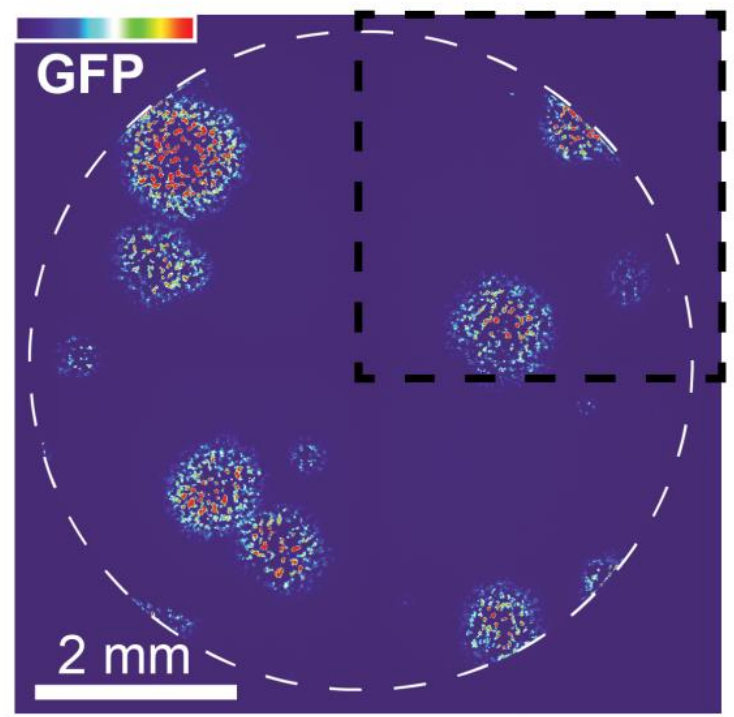

d

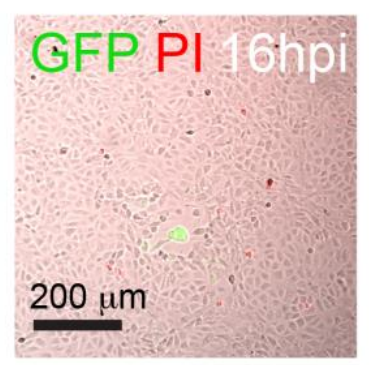

b
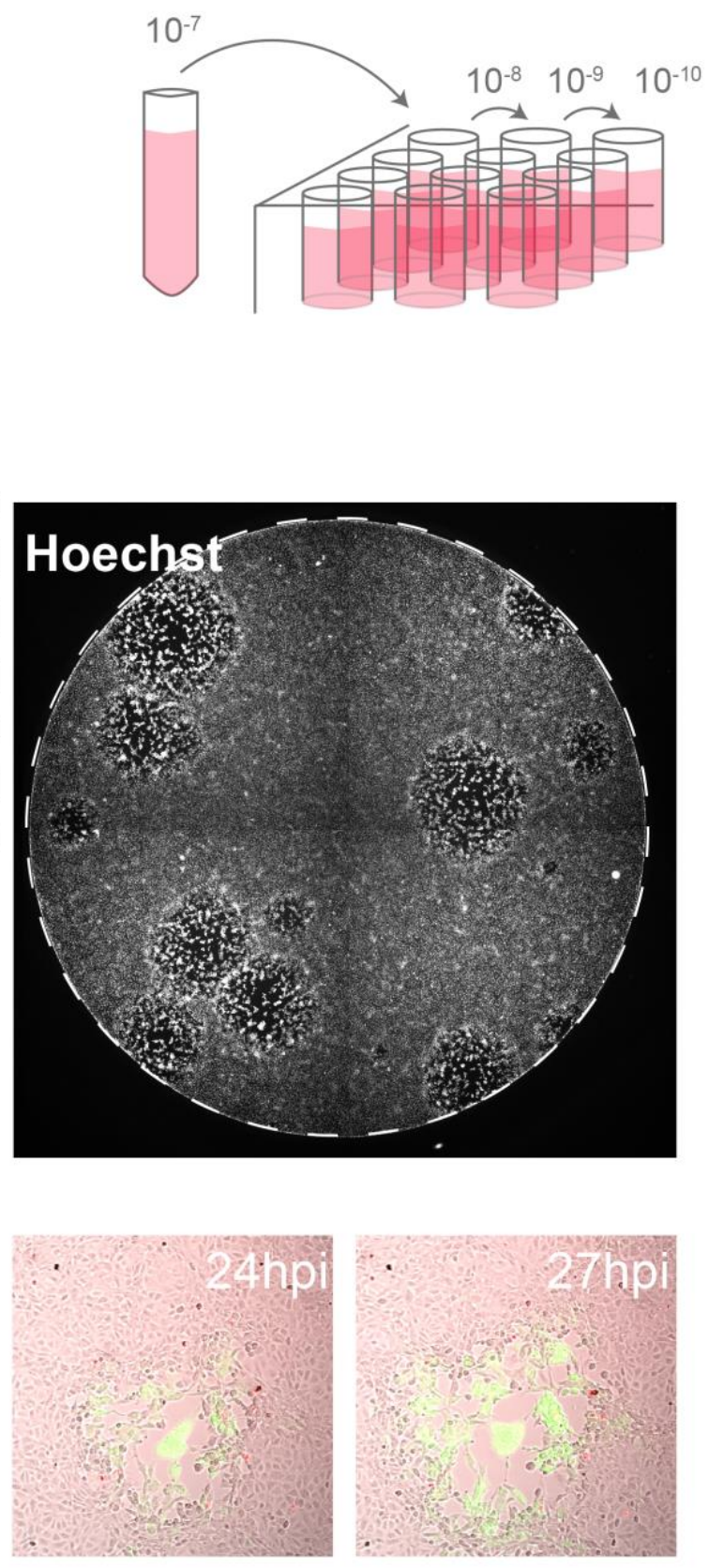

Fig. 1. Principles of the High-Content VACV Plaque Assay. (a) Schematic representation of the serial pre-dilution prior to inoculum dispensing into the plate. (b) Schematic representation of serial dilution performed directly in the plate. (c) A typical example of High-content-fluorescence-microscopy-based plaque assay (Plaque2.0) performed as an end point assay using VACV WR E/L EGFP virus. On the left-hand 
side, image shows virus EGFP signal intensity is color-coded violet (minimum) to red (maximum). On the right-hand side, image shows Hoechst signal from the monolayer nuclei. (d) Typical images from High-content-fluorescence-microscopy-based plaque assay performed in live cell imaging setting. Still frames of merged transmission light, propidium iodide (PI, red) and VACV IHD-J E/L EGFP (GFP, green) signals are arranged in a time dependent manner showing dynamics of the growing VACV plaque. Here PI signal is used to indicate monolayer cells death. Time points are indicated as hours post infection (hpi). 
a

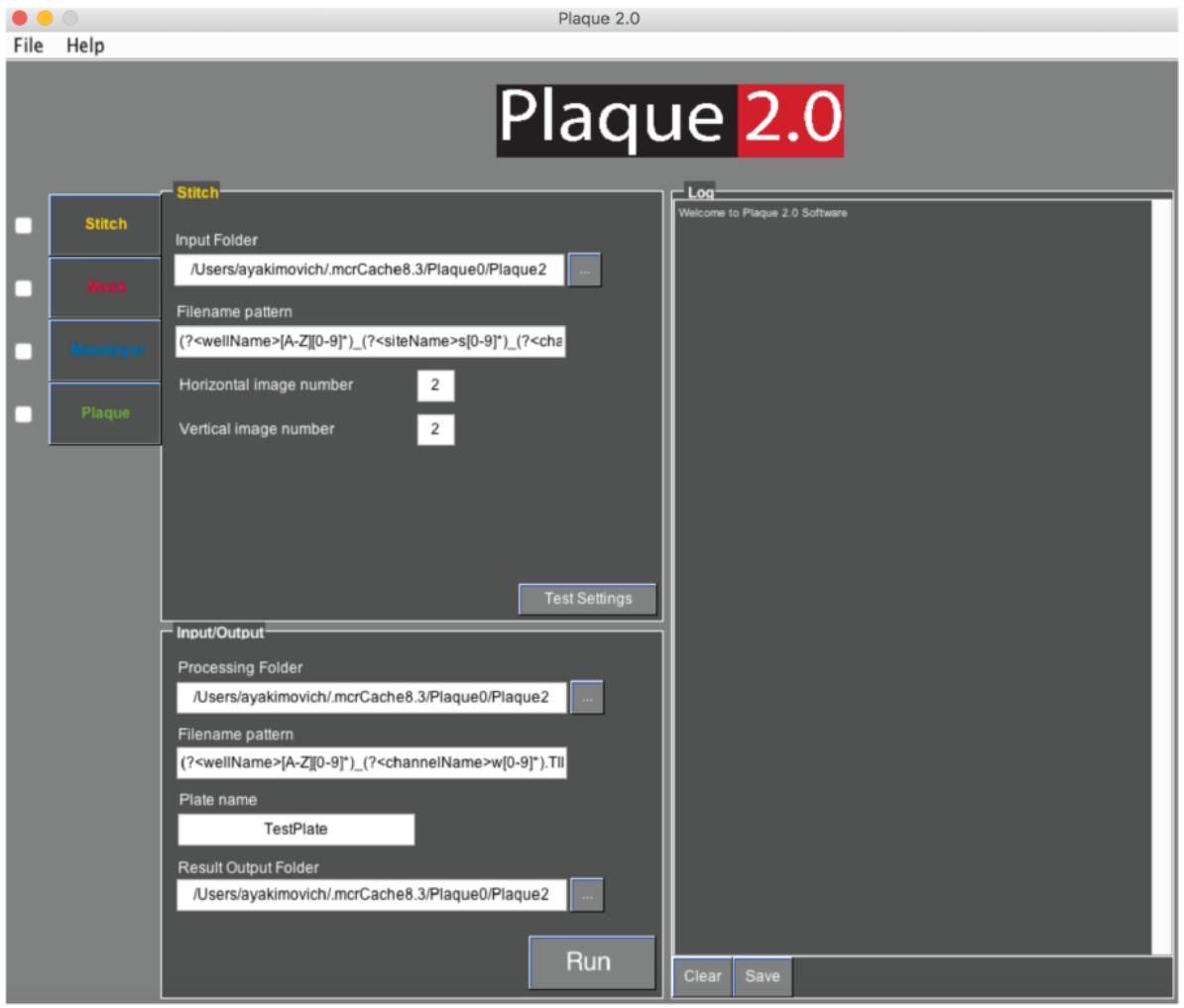

b

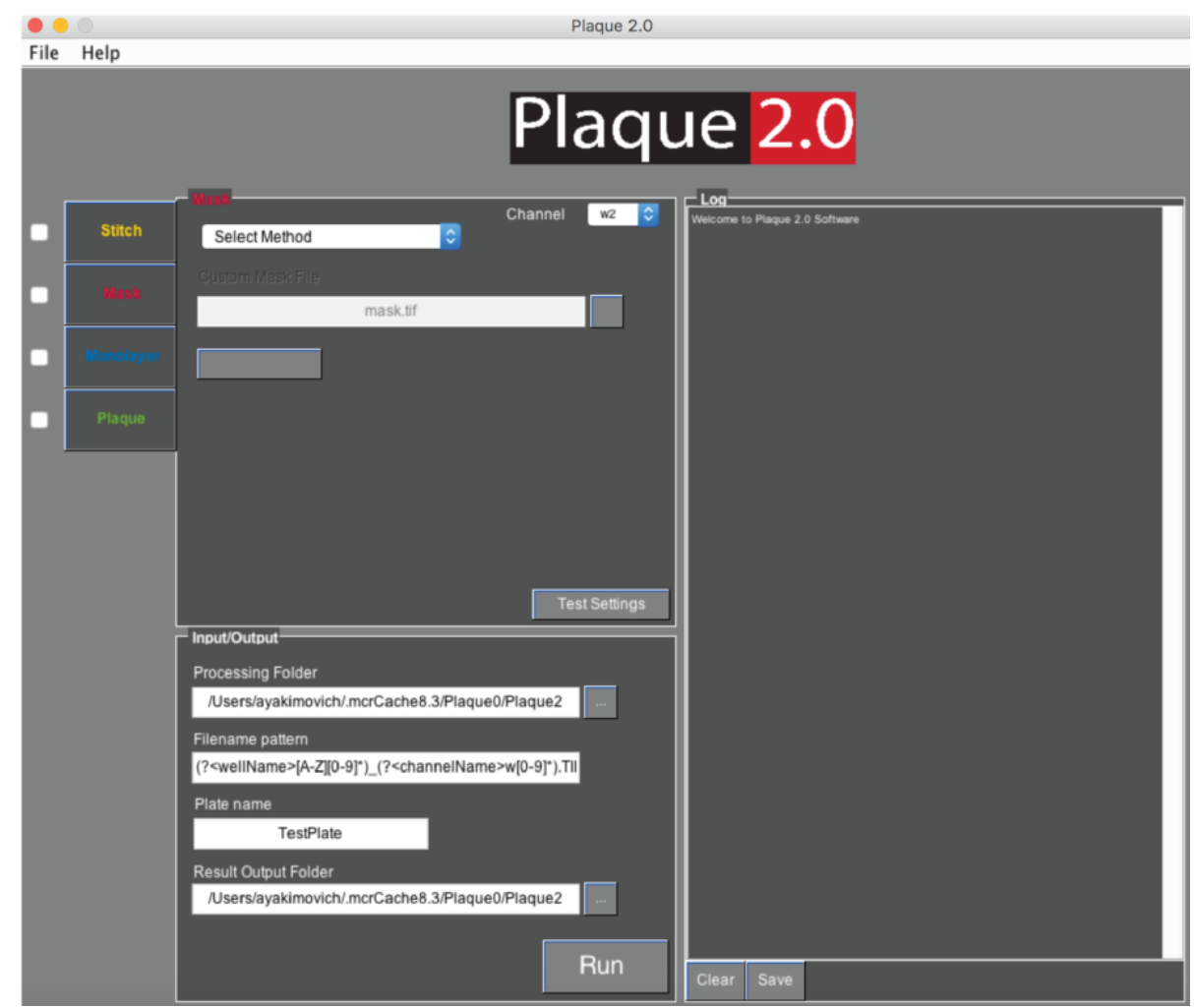

Fig. 2. Plaque2.0 Software interface view showing the main window and the respective activated pane. (a) View of the user defined parameters in the "Stich" pane. (b) View of the user defined parameters in the "Mask" pane. 
a

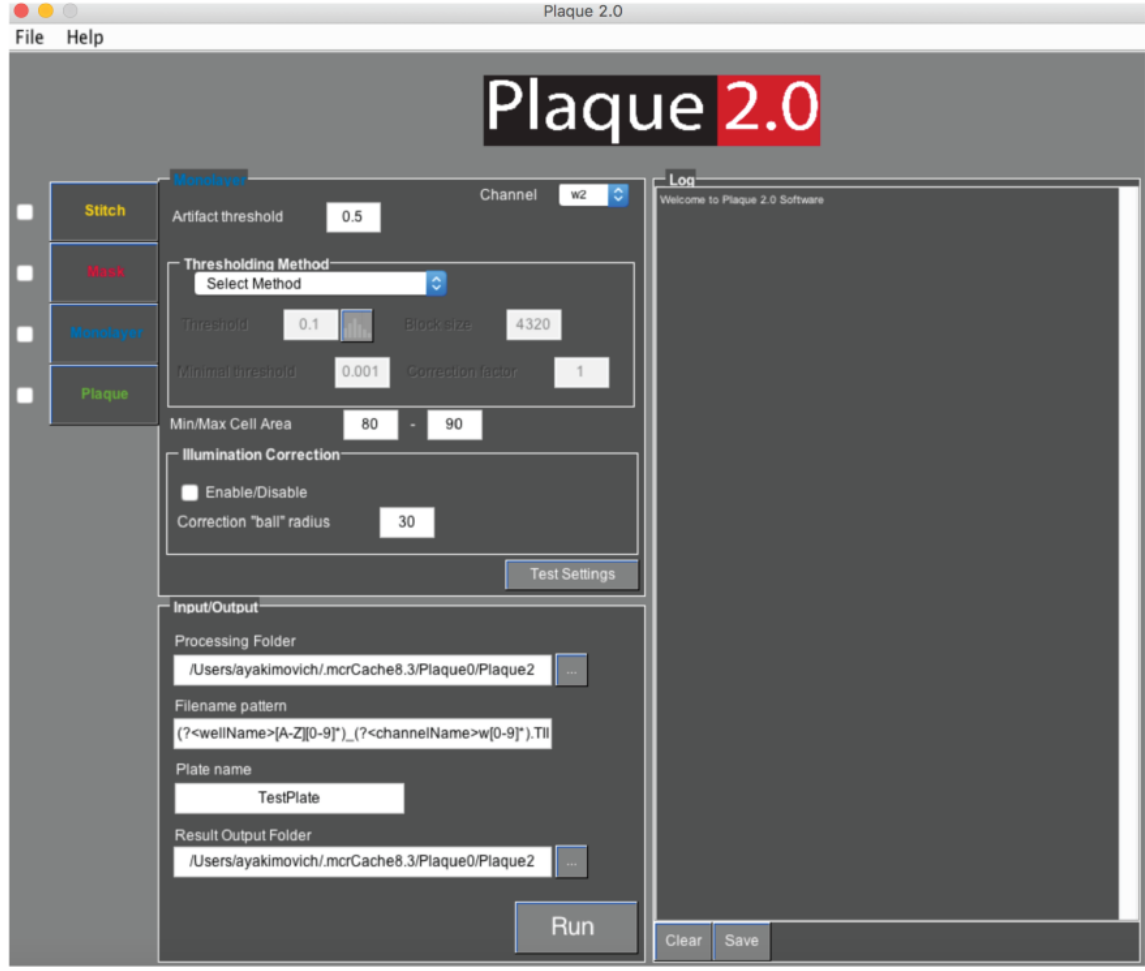

b

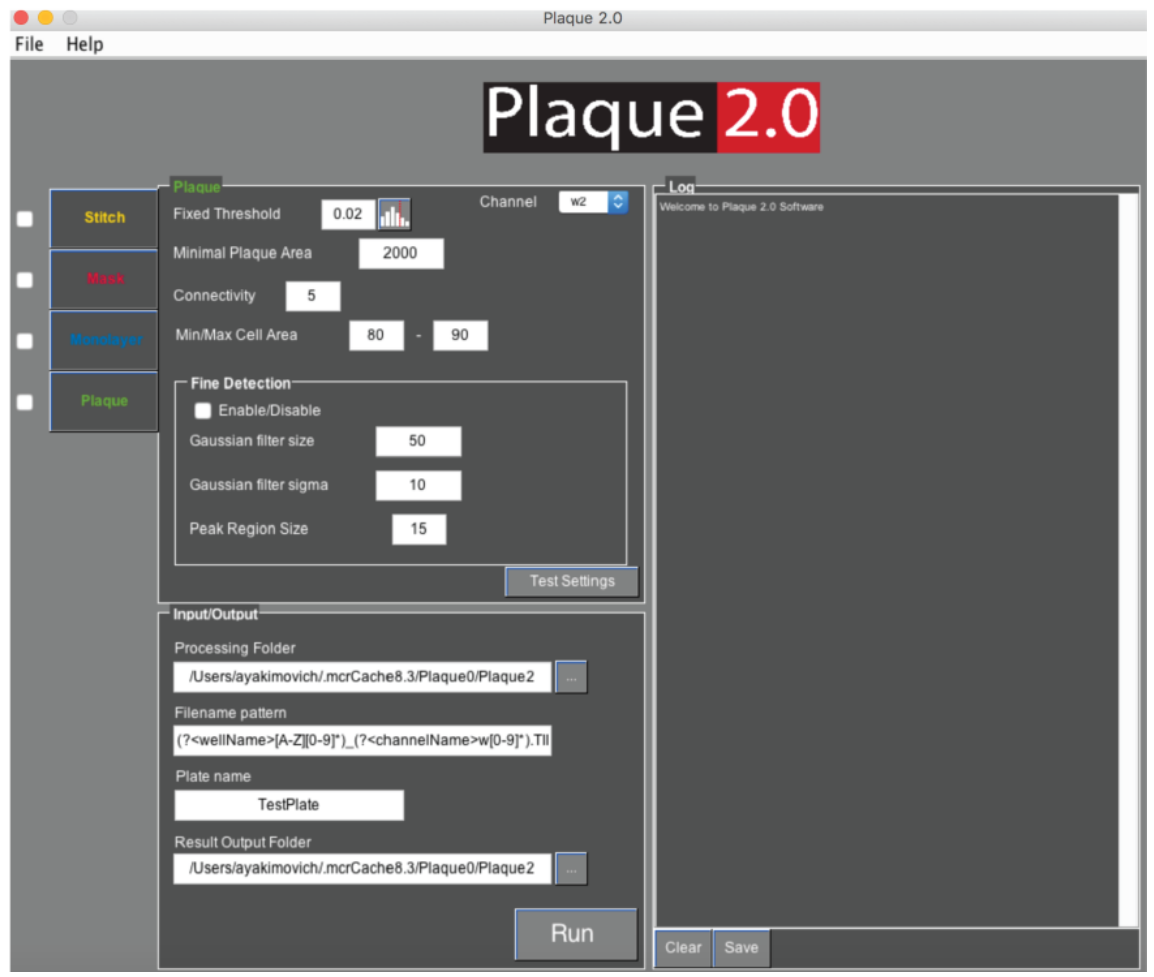

Fig. 3. Plaque2.0 Software interface view showing the main window and the respective activated pane. (a) View of the user defined parameters in the "Monolayer" pane. (b) View of the user defined parameters in the "Plaque" pane. 
Table 1: Detailed overview of all user-defined parameters in the Plaque2.0 software.

\begin{tabular}{|c|c|}
\hline \multicolumn{2}{|c|}{ Main Parameters (Input/Output) } \\
\hline Parameter & Description \\
\hline Processing Folder & $\begin{array}{l}\text { Use to indicate the location of microscopy images that do } \\
\text { not require stitching or have been processed by the } \\
\text { "Stitch" module. }\end{array}$ \\
\hline Filename pattern & $\begin{array}{l}\text { This input defines the regular expression (RegEx) that is } \\
\text { used to parse the metadata from the image filenames (well } \\
\text { rows, well columns, channels). Metadata will be use to } \\
\text { formulate readouts. }\end{array}$ \\
\hline Plate name & $\begin{array}{l}\text { This input defines a name for the current plate analyzed. } \\
\text { The parameter is used as a prefix for output files. }\end{array}$ \\
\hline Result Output Folder & Defines location of the output files. \\
\hline \multicolumn{2}{|r|}{ Stich Window } \\
\hline Parameter & Description \\
\hline Input Folder & $\begin{array}{l}\text { Use to indicate the location of the microscopy images to } \\
\text { be stitched by this module. }\end{array}$ \\
\hline Filename pattern & $\begin{array}{l}\text { This input defines the RegEx used to parse the metadata } \\
\text { (well rows, well columns, channels, sites) from the } \\
\text { filenames of the images. }\end{array}$ \\
\hline $\begin{array}{l}\text { Horizontal Image Number, } \\
\text { Vertical Image Number }\end{array}$ & $\begin{array}{l}\text { This input specifies the number of well sites for full well } \\
\text { stitching in horizontal and vertical direction accordingly. }\end{array}$ \\
\hline \multicolumn{2}{|r|}{ Mask Window } \\
\hline Parameter & Description \\
\hline Load Custom Mask & $\begin{array}{l}\text { If this mask definition method is selected the well mask is } \\
\text { loaded from the file provided by the input path. }\end{array}$ \\
\hline Manual Mask Definition & $\begin{array}{l}\text { If this mask definition method is selected the "Define } \\
\text { Mask" button becomes enabled. Upon pressing the latter, } \\
\text { a new window where the user can specify the mask for the } \\
\text { plate wells is opened. This is done by drag re-sizing an oval } \\
\text { outline over the first stitched image from the "Processing } \\
\text { Folder". The mask determined is then saved in the current } \\
\text { working folder by double clicking the image. }\end{array}$ \\
\hline Automatic Mask Definition & $\begin{array}{l}\text { If this mask definition method is selected, the mask is } \\
\text { determined automatically using the first stitched image } \\
\text { from the "Processing Folder". }\end{array}$ \\
\hline \multicolumn{2}{|r|}{ Monolayer Window } \\
\hline Parameter & Description \\
\hline Artifact threshold & $\begin{array}{l}\text { This setting defines an upper threshold (grayscale values } \\
\text { stretched between } 0 \text { and } 1 \text { ) used to filter out very bright } \\
\text { imaging artifacts. }\end{array}$ \\
\hline Manual Thresholding & $\begin{array}{l}\text { If method is selected for thresholding a manual user- } \\
\text { defined value will be used to perform foreground } \\
\text { segmentation. }\end{array}$ \\
\hline Otsu Global Thresholding & $\begin{array}{l}\text { Selecting this method will perform automatic Otsu gloabl } \\
\text { thresholding based segmentation of foreground pixels. }\end{array}$ \\
\hline
\end{tabular}




\begin{tabular}{|c|c|}
\hline Otsu Local Thresholding & $\begin{array}{l}\text { Selecting this method will perform automatic Otsu local } \\
\text { thresholding based segmentation of foreground pixels. } \\
\text { Here the image is split into smaller blocks. Otsu } \\
\text { thresholding is performed separately for each of the blocks } \\
\text { allowing local threshold values to be used. }\end{array}$ \\
\hline Threshold & $\begin{array}{l}\text { This value specifies the manual threshold (defined } \\
\text { between } 0 \text { and } 1 \text { ) for Manual Thresholding. }\end{array}$ \\
\hline Block size & $\begin{array}{l}\text { This input provides one size (one dimension) of a square } \\
\text { block side (pixels) to be used for the "Otsu Local } \\
\text { Thresholding" method. Note, that width or height of the } \\
\text { image should be divisible by the "Block size". }\end{array}$ \\
\hline Minimal threshold & $\begin{array}{l}\text { This value (between } 0 \text { and } 1 \text { ) defines the lowest threshold } \\
\text { possible for the "Otsu Global Thresholding". }\end{array}$ \\
\hline Correction factor & $\begin{array}{l}\text { Here a threshold correction factor (between } 0 \text { and } 1-\text { to } \\
\text { decrease, higher than } 1 \text { to increase) can be defined to fine- } \\
\text { tune the results of the "Otsu Global Thresholding". }\end{array}$ \\
\hline Min/Max Cell Area & $\begin{array}{l}\text { These values are used for cell number calculation. Specify } \\
\text { minimum and maximum nucleus area. }\end{array}$ \\
\hline Correction "ball" radius & $\begin{array}{l}\text { If used, this value provides the radius of a, so called, } \\
\text { "rolling ball", used in the "Rolling Ball" algorithm for the } \\
\text { illumination correction. Radius should be lower, than the } \\
\text { typical size of an object in the image. }\end{array}$ \\
\hline \multicolumn{2}{|r|}{ Plaque Window } \\
\hline Parameter & Description \\
\hline Fixed Threshold & $\begin{array}{l}\text { This value (between } 0 \text { and } 1 \text { ) defines a fixed manual } \\
\text { threshold for virus image foreground detection. }\end{array}$ \\
\hline Minimal Plaque Area & $\begin{array}{l}\text { Here the minimum area of a detected object to be } \\
\text { considered a plaque is specified. }\end{array}$ \\
\hline Connectivity & $\begin{array}{l}\text { This input defines the maximum distance plaque regions } \\
\text { (pixels). }\end{array}$ \\
\hline Min/Max Cell Area & $\begin{array}{l}\text { This value defines minimum and maximum cellular area in } \\
\text { the virus signal image for cell number calculation. This } \\
\text { parameter is used only when the cell number estimation } \\
\text { based on the nuclear segmentation is disabled. }\end{array}$ \\
\hline Gaussian filter size & $\begin{array}{l}\text { This input defines the of the "blurring bell"(Gaussian filter) } \\
\text { for virus image convolution (pixels). It is used for detecting } \\
\text { plaques. }\end{array}$ \\
\hline Gaussian filter sigma & $\begin{array}{l}\text { Here the width of the "blurring bell" (standard deviation of } \\
\text { the Gaussian filter) for virus image convolution is defined. } \\
\text { It is used for detecting plaques together with the previous } \\
\text { parameter. }\end{array}$ \\
\hline Peak Region Size & $\begin{array}{l}\text { This input defines the size of a plaque region (maximum) in } \\
\text { pixels. It is assumed that only one intensity maximum can } \\
\text { be detected per plaque. }\end{array}$ \\
\hline
\end{tabular}


Parameter column represents the parameter name in the software. Description software represents details about the specific parameter. Section headers correspond to the respective name of the pane. 
Table 2. Detailed overview of all readouts provided by the Plaque2.0 software in the resulting comma separated value files.

\begin{tabular}{|c|c|}
\hline \multicolumn{2}{|r|}{ Image-based Features } \\
\hline Feature & Description \\
\hline NucleilmageName & Measured file name (nuclei signal image). \\
\hline wellRow & Measured plate row. \\
\hline wellCollumn & Measured plate column. \\
\hline maxNucleilntensity & Nuclear intensity maximum in the image measured. \\
\hline totalNucleilntensity & $\begin{array}{l}\text { Nuclear Intensities sum in the image measured (after } \\
\text { masking). }\end{array}$ \\
\hline meanNucleilntensity & $\begin{array}{l}\text { Nuclear Intensities mean in the image measured (after } \\
\text { masking). }\end{array}$ \\
\hline numberOfNuclei & Number of nuclei estimated per image. \\
\hline VirusImageName & Measured file name (virus signal image). \\
\hline maxVirusIntensity & $\begin{array}{l}\text { Virus Intensities maximum in the image measured } \\
\text { (after masking). }\end{array}$ \\
\hline totalVirusIntensity & $\begin{array}{l}\text { Virus Intensities sum in the image measured (after } \\
\text { masking). }\end{array}$ \\
\hline meanVirusIntensity & $\begin{array}{l}\text { Virus Intensities mean in the image measured (after } \\
\text { masking). }\end{array}$ \\
\hline numberOfPlaques & Number of detected plaques per image. \\
\hline numberOfInfectedNuclei & $\begin{array}{l}\text { Number of infected nuclei detected after overlaying } \\
\text { the nuclear image (if applicable) with thresholder virus } \\
\text { image. If no nuclear image is provided, virus image is } \\
\text { used for the estimation. }\end{array}$ \\
\hline \multicolumn{2}{|r|}{ Plaque-based Features } \\
\hline Feature & Description \\
\hline Area & Area of the plaque region (pixel). \\
\hline Centroid & Centroid position of the plaque region. \\
\hline BoundingBox & $\begin{array}{l}\text { Coordinates of the plaque region in boundaries } \\
\text { provided in the following format: } x y \text { width height. }\end{array}$ \\
\hline MajorAxisLength & $\begin{array}{l}\text { Size of the major axis of the fitted ellipsoid to the } \\
\text { measured plaque region. }\end{array}$ \\
\hline MinorAxisLength & $\begin{array}{l}\text { Size of the minor axis of the fitted ellipsoid to the } \\
\text { measured plaque region. }\end{array}$ \\
\hline Eccentricity & $\begin{array}{l}\text { Ellipsoid eccentricity - i.e. how much the ellipse fitted } \\
\text { to the measured plaque region varies from being } \\
\text { circular. }\end{array}$ \\
\hline ConvexArea & Convex area of the measured plaque region. \\
\hline numberOfNucleilnPlaque & Nuclei number estimated in the plaque. \\
\hline numberOfInfectedNucleilnPlaque & Infected nuclei number estimated in the plaque. \\
\hline wellRow & Measured plate row. \\
\hline wellCollumn & Measured plate column. \\
\hline
\end{tabular}




\begin{tabular}{|l|l|}
\hline maxIntensityGFP & $\begin{array}{l}\text { Virus Intensities maximum in the image measured } \\
\text { (after masking). }\end{array}$ \\
\hline totallntensityGFP & $\begin{array}{l}\text { Virus Intensities sum in the image measured (after } \\
\text { masking). }\end{array}$ \\
\hline meanIntensity & $\begin{array}{l}\text { Virus Intensities mean in the image measured (after } \\
\text { masking). }\end{array}$ \\
\hline
\end{tabular}

Section headers correspond to the respective readout group (image-based or objectbased) saved as a single file per group. 